\title{
Assessment of Phenotype Microarray plates for rapid and high-throughput analysis of collateral sensitivity networks
}

Elsie J. Dunkley, James D. Chalmers, Stephanie Cho, Thomas J. Finn, Wayne M. Patrick

\section{Supporting Information}

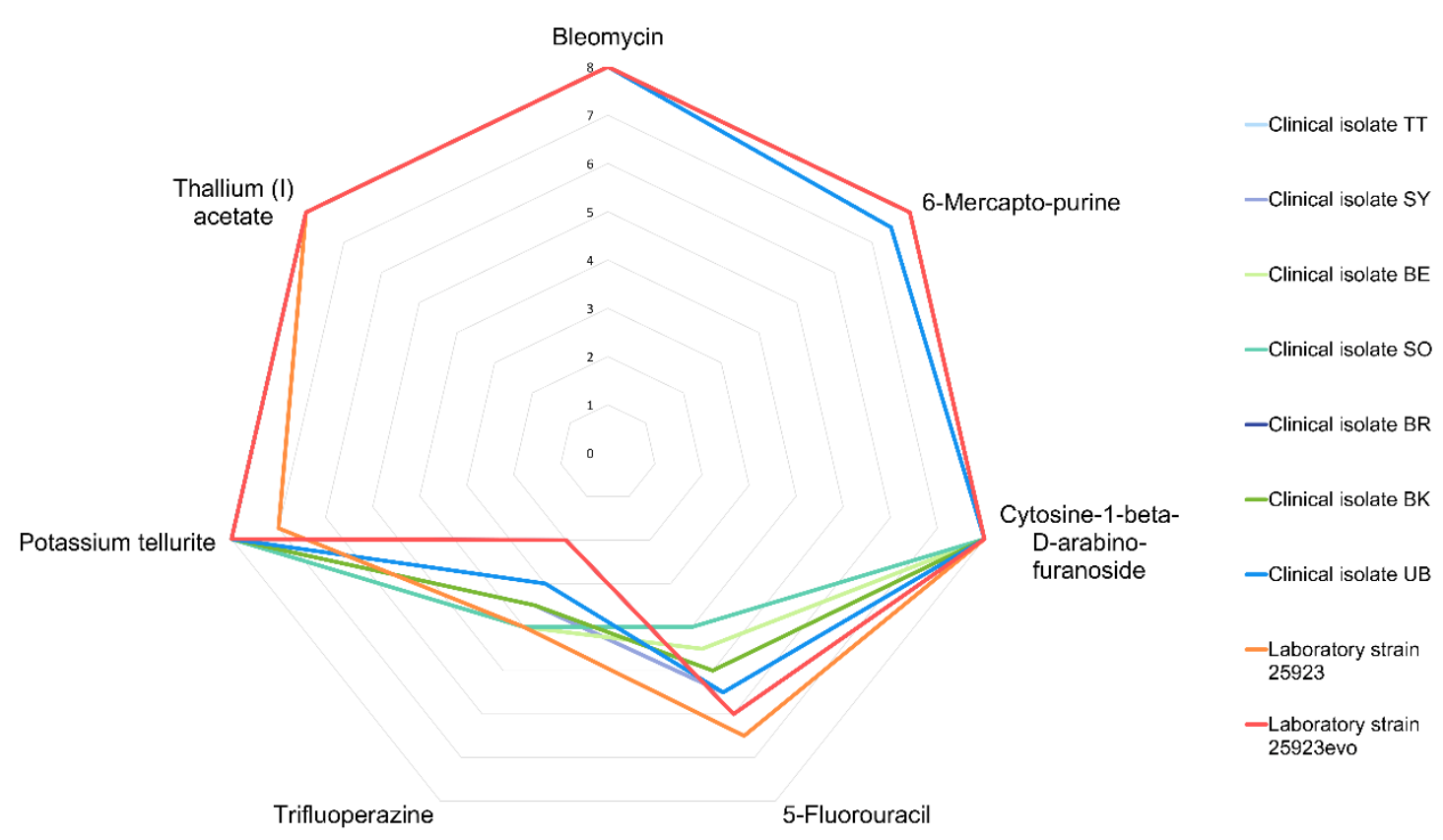

S4 Fig. Phenotype Microarray scores for nine $S$. aureus strains in the presence of repurposed cancer and antipsychotic drugs. Scores are an average of two biological replicates, with 8 representing maximum relative resistance around the exterior of the radar and 0 representing complete sensitivity at the centre. Clinical isolates are in shades of blue and green. Laboratory strains S. aureus ATCC 25923 and 25923evo are orange and red respectively. 